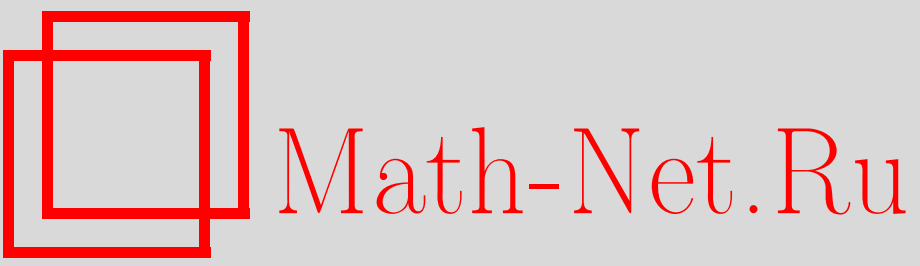

С. Г. Глебов, Резонансные слои в задачах о взаимодействии быстроосциллирующих волновых полей, ТМФ, 1999, том 118, номер 3, 375-382

DOI: https://doi.org/10.4213/tmf709

Использование Общероссийского математического портала Math-Net.Ru подразумевает, что вы прочитали и согласны с пользовательским соглашением

http://www.mathnet.ru/rus/agreement

Параметры загрузки:

IP: 52.90.164.192

26 апреля 2023 г., 11:46:02 


\section{РЕЗОНАНСНЫЕ СЛОИ В ЗАДАЧАХ О ВЗАИМОДЕЙСТВИИ \\ БЫСТРООСЦИЛЛИРУЮЩИХ ВОЛНОВЫХ ПОЛЕЙ}

Изучаются явления, имеющие место при слабом резонансном взаимодействии быстроосциллирующих волновых полей. В общем случае при резонансном взаимодействии обнаруживаются узкие переходные слои, внутри которых динамика волнового поля описывается в терминах нелинейного уравнения Шредингера.

Целью данной работы является описание динамики главного члена формального асимптотического решения задачи о слабом нелинейном взаимодействии в узких переходных слоях вблизи резонанса.

Известно, что многие волновые уравнения допускают решения в виде плоской, монохроматической волны $U=A \exp \{i(\langle k, x\rangle-\omega t)\}, k \in \mathbb{R}^{n}, x \in \mathbb{R}^{n}$. При этом частота $\omega$ и волновой вектор $k$ не произвольны, а связаны дисперсионным соотношением $L(\omega, k)=0$. Вид дисперсионного соотношения определяется выбором уравнения. Однако большая часть решений не допускает выражения через столь простые функции. Поэтому имеет смысл искать решения не такого вида, а близкие к ним. Мерой близости является малый параметр $0<\varepsilon \ll 1$.

В математической постановке задачи малый параметр может явно входить в начальные данные, характеризуя их отклонение от невозмушенных начальных данных. Примером служат начальные данные с медленной деформацией фазы $A \exp \left\{i S_{0}(\varepsilon x) / \varepsilon\right\}$ или амплитуды $A(\varepsilon x) \exp \{i\langle k, x\rangle\}$. Обычно такие задачи записываются в "медленных" переменных $\xi=\varepsilon x, \theta=\varepsilon t$ так, что соответствуюшее дифференциальное уравнение содержит малые множители при производных,

$$
L\left(i \varepsilon \partial_{\theta},-i \varepsilon \nabla_{\xi}\right) U=0,\left.\quad U\right|_{\theta=0}=A(\xi) \exp \left\{i \frac{S_{0}(\xi)}{\varepsilon}\right\} .
$$

Асимптотические решения таких задач хорошо известны и описываются формулами ВКБ-приближений или коротковолновой асимптотики

$$
U=\exp \left\{i \frac{S(\xi, \theta)}{\varepsilon}\right\} \sum_{n \geqslant 0} \varepsilon^{n} \stackrel{n}{A}(\xi, \theta)
$$

* Уфимский государственный неффяной технический университет, Уфа, Россия. E-mail: gloomy@imat.rb.ru; gloomy@oil.ru 
Фаза $S$ определяется из уравнения эйконала $L\left(-\partial_{\theta} S, \nabla_{\xi} S\right)=0$, а коэффициенты разложения амплитуды $\stackrel{n}{A}$ - из уравнений переноса.

Эти формулы дают асимптотическое решение для линейных уравнений. Линейность обеспечивает простую суперпозицию волн с разными фазами. Такая ситуация сохраняется и при возмушении дифференшиального уравнения

$$
L\left(i \varepsilon \partial_{\theta},-i \varepsilon \nabla_{\xi}, \xi, \theta ; \varepsilon\right) U=\varepsilon f(U, \xi, \theta),
$$

если возмушаюшие добавки - линейные операторы. При этом в асимптотическом решении изменения касаются лишь уравнений переноса.

Иное дело, если берется нелинейное возмушение. В этом случае возможны совершенно иные эффекты. Уравнения для амплитуд становятся квазилинейными и возможно появление в главном члене асимптотического решения слагаемых с новыми комбинационными фазами. Подобные ситуации характеризуются термином "резонансные". В случае фаз с медленной деформацией $S_{1}, S_{2}$, которые определяются из уравнений эйконала, резонансными называют такие ситуации, в которых комбинационная фаза $S(\xi, \theta)=S_{1}+S_{2}$ сама удовлетворяет уравнению эйконала. Для разных типов решений уравнение эйконала может вьполняться тождественно по $(\xi, \theta) \in \mathbb{R}^{n+1}$ (глобальный резонанс) либо на многообразиях меньшей чем $n+1$ размерности (слабый или локальный резонанс).

Задачи с локальным резонансом рассматривались как для обыкновенных дифференциальных уравнений [1-3], так и для систем уравнений в частных производных [4-7]. В данной статье рассматривается последний случай. Оказывается, что вблизи локального резонанса становится непригодным анзац ВКБ (1). Для того чтобы правильно описать поведение решения в окрестности резонансных многообразий (в переходных слоях), вводятся новые "растянутые" переменные, а затем с использованием этих новых переменных в слое локального резонанса строится свой "внутренний" анзац. При этом, естественно, требуется согласованность "внешнего" и "внутреннего" анзацев.

\section{1. ВОЗМУЩЕНИЕ ВОЛНОВОГО УРАВНЕНИЯ}

Рассмотрим для иллюстрации задачу о возмушении волнового уравнения известной быстроосциллирующей силой

$$
\varepsilon^{2} U_{\theta \theta}-\varepsilon^{2} c^{2}(\xi, \theta) U_{\xi \xi}=\exp \left\{i \frac{\Phi(\xi, \theta)}{\varepsilon}\right\}, \quad 0<\varepsilon \ll 1 .
$$

Фаза возмушения $\Phi$ в уравнении (2) - известная гладкая функция своих переменных.

Будем искать формальное асимптотическое решение (ФАР) уравнения (2) в виде ВКБ-анзаца

$$
\left.U(\xi, \theta ; \varepsilon)=\exp \left\{i \frac{\Phi(\xi, \theta)}{\varepsilon}\right\}[0] U(\xi, \theta)+\varepsilon U^{0}(\xi, \theta)+\cdots\right] .
$$

Подставляя анзац (3) в уравнение (2) и собирая слагаемые при одинаковых степенях малого параметра, получаем рекуррентную последовательность уравнений для определения амплитуд $\stackrel{n}{U}(\xi, \theta)$. 
Главный член $\Phi$ АР

$$
\stackrel{0}{U}=\frac{1}{c^{2} \Phi_{\xi}^{2}-\Phi_{\theta}^{2}}
$$

имеет особенность в пространстве медленных переменных $(\xi, \theta)$ около кривой, где волновое число $\Phi_{\xi}$ и частота $\Phi_{\theta}$ возмушения удовлетворяют уравнению эйконала

$$
S_{\theta}^{2}-c^{2} S_{\xi}^{2}=0
$$

В следующих поправках порядок особенности нарастает. Ряд (3) перестает быть асимптотическим в узкой (порядка $\sqrt{\varepsilon}$ ) окрестности этой кривой. Это указывает на изменение характера главного члена формального асимптотического решения вблизи резонанса.

Выберем какую-нибудь одну из кривых $\Phi_{\theta} \pm c \Phi_{\xi}=0$, например $l_{+}$, и проведем построения в ее окрестности. Построения около резонансной кривой $l_{-}$проводятся аналогично.

Введем характерную переменную $Z=\Phi_{\theta}+c \Phi_{\xi}$, которая может рассматриваться как расстояние до кривой $l_{+}$. Задача описания поведения решения при подходе к резонансной кривой равносильна построению $\Phi$ АР при $Z \rightarrow 0$. ФАР уравнения $(2)$ при подходе к линии локального резонанса (при $Z \rightarrow 0$ ) имеет вид

$$
U(Z, \theta ; \varepsilon)=\sum_{n \geqslant 0} \frac{\varepsilon^{n}}{Z^{2 n+1}} \stackrel{n}{\widetilde{U}}(Z, \theta), \quad Z \rightarrow 0,
$$

здесь амплитуды $\stackrel{n}{\widetilde{U}}$ - гладкие функции по переменной $Z$.

Для того чтобы правильно описать решение в окрестности кривой $l_{+}$, введем новую "растянутую" переменную $\zeta=Z / \sqrt{\varepsilon}$.

Внутреннее ФАР будем искать в виде

$$
U(\xi, \theta, \zeta ; \varepsilon)=e^{i \frac{\Psi(\xi, \theta)}{\varepsilon}}\left[\varepsilon^{-1 / 2} \frac{-1}{V}(\zeta, \theta)+\stackrel{0}{V}(\zeta, \theta)+\sqrt{\varepsilon} \stackrel{1}{V}(\zeta, \theta)+\cdots\right],
$$

здесь фаза $\Psi$ внутреннего решения удовлетворяет уравнению эйконала $\Psi_{\theta}+c \Psi_{\xi}=0$ и условиям $\Psi=\Phi, \Psi_{\theta}=\Phi_{\theta}, \Psi_{\xi}=\Phi_{\xi}$ на резонансной кривой $l_{+}$. Появление отрицательных степеней малого параметра $\varepsilon$ в асимптотическом решении (4) обусловлено резонансом и соответствует особенностям во внешнем разложении.

Для амплитуды главного члена асимптотики внутреннего решения получаем неоднородное линейное уравнение Шредингера

$$
i \partial_{\theta} \bar{V}^{-1}+a(\theta) \partial_{\zeta}^{2} \bar{V}+i b(\theta) \stackrel{-1}{V}=c(\theta) \exp \left\{i p_{0}(\theta) \zeta^{2}\right\}
$$

коэффициенты которого - известные функции, зависяшие только от переменной $\theta$. Из условия согласования с решением во внешней области для функции $\stackrel{-1}{V}$ выписывается асимптотика при $|\zeta| \rightarrow \infty:$

$$
\left.\bar{V}\right|_{|\zeta| \rightarrow \infty} \sim O\left(\frac{1}{|\zeta|}\right) .
$$




\section{2. ЗАДАЧА КОШИ ДЛЯ СЛАБОНЕЛИНЕЙНОГО УРАВНЕНИЯ ШРЕДИНГЕРА}

Основной результат статьи содержится в этом разделе. Рассмотрим задачу Коши для двумерного слабонелинейного уравнения Шредингера

$$
i \varepsilon \partial_{\theta} U+\varepsilon^{2} \Delta U+q U+\varepsilon \gamma|U|^{2} U=0,
$$

где $\Delta=\partial_{\xi}^{2}+\partial_{\eta}^{2}, 0<\varepsilon \ll 1, q(\xi, \eta, \theta)$ - известная гладкая функция.

Начальные данные берутся в виде суммы двух быстроосциллируюших гармоник с медленно меняющимися амплитудами

$$
\left.U\right|_{\theta=0}=A_{1}(\xi, \eta) \exp \left(i \varepsilon^{-1} \stackrel{0}{\varphi}_{1}\right)+A_{2}(\xi, \eta) \exp \left(i \varepsilon^{-1} \stackrel{0}{\varphi}_{2}\right) .
$$

Будем предполагать, что исходные фазы $\stackrel{0}{\varphi}_{1}, \stackrel{0}{\varphi}_{2}$ в начальный момент не произвольны, а связаны соотношением

$$
\nabla \stackrel{\varphi}{\varphi}_{1}(\xi, \eta)=\nabla \stackrel{0}{\varphi}_{2}(\xi, \eta)
$$

в одной изолированной точке $\left(\xi_{0}, \eta_{0}\right)$.

Условия (7) замечательны тем, что они приводят к появлению в пространстве независимых переменных резонансных многообразий. Многообразием локального резонанса оказывается одномерная кривая, образуемая совпадаюшими лучами, выходяшими из точки $\left(\xi_{0}, \eta_{0}\right)$. Под лучами здесь понимаются характеристики уравнения эйконала для соответствуюшей фазы.

Наша цель состоит в построении формального асимптотического решения задачи (5), (6), равномерно пригодного в некотором слое $\Omega=\left\{(\xi, \eta) \in \mathbb{R}^{2}, 0 \leqslant \theta \leqslant \theta^{*}\right\}$. При этом, как обычно [8], вся область $\Omega$ разбивается на несколько подобластей со своим представлением решения в каждой из них. Подобласти удобно определить в переменных $S=\sqrt{2} \partial_{\xi}\left(\varphi_{1}-\varphi_{2}\right)$ и $R=\sqrt{2} \partial_{\eta}\left(\varphi_{1}-\varphi_{2}\right)$,

$$
\begin{aligned}
& \Omega_{\mathrm{ex}}=\left\{0 \leqslant \theta \leqslant \theta^{*}, \sqrt{S^{2}+R^{2}} \geqslant \varepsilon^{1 / 4}\right\} \text { - внешняя область, } \\
& \Omega_{\mathrm{in}}=\left\{0 \leqslant \theta \leqslant \theta^{*}, \sqrt{S^{2}+R^{2}} \leqslant 2 \varepsilon^{1 / 4}\right\} \text { - внутренняя область. }
\end{aligned}
$$

Справедлива следуюшая теорема.

Теорема 1. Пусть функции $A_{k}, \stackrel{0}{\varphi} k$ и любые их производные равномерно ограничены по переменным $\xi, \eta$. Тогда в области $\Omega$ формальное асимптотическое при $\varepsilon \rightarrow 0$ решение задачи (5), (6) по модулю $O\left(\varepsilon^{5 / 2}\right)$ в различных областях имеет разное асимптотическое представление,

$$
U(\xi, \eta, \theta ; \varepsilon) \approx U_{0}+\varepsilon U_{1}+\varepsilon^{2} U_{2}, \quad(\xi, \eta, \theta) \in \Omega_{\mathrm{ex}},
$$

$u$

$$
U(\xi, \eta, \theta ; \varepsilon) \approx \exp \left\{i \varepsilon^{-1} \varphi_{1}\right\}\left[{ }^{0}+\sqrt{\varepsilon} \stackrel{1}{V}+\varepsilon V^{2}\right], \quad(\xi, \eta, \theta) \in \Omega_{\text {in }}
$$


Построение $\Phi$ АР во внешней области не вызывает затруднений. Решение ишется в виде обычного ВКБ-анзаца (8), где

$$
U_{n}(\xi, \eta, \theta ; \varepsilon)=\left[\sum_{\varphi \in \Phi_{n}} \stackrel{n}{A}_{\varphi} \exp \left(i \varepsilon^{-1} \varphi\right)+\sum_{\psi \in \Psi_{n}} \stackrel{n}{B}_{\psi} \exp \left(i \varepsilon^{-1} \psi\right)\right],
$$

неизвестные функции $\stackrel{n}{A}_{\varphi}, \stackrel{n}{B},, \varphi, \psi$ зависят от переменных $(\xi, \eta, \theta)$. Суммирование в формуле (10) происходит как по собственным фазам $\varphi \in \Phi_{n}, n=0,1, \ldots$, удовлетворяющим уравнению эйконала, так и по несобственным комбинационным фазам $\psi \in \Psi_{n}$, $n=0,1, \ldots$ В каждой поправке $U_{n}$ имеется только конечное число слагаемых.

После подстановки представления (8) в задачу $(5),(6)$ получается рекуррентная последовательность задач для определения фаз и амплитуд.

На первом шаге определяются собственные фазы $\varphi_{1}, \varphi_{2}$. Они удовлетворяют неоднородному уравнению эйконала

$$
l\left[\varphi_{k}\right] \equiv \partial_{\theta} \varphi_{k}+\left(\partial_{\xi} \varphi_{k}\right)^{2}+\left(\partial_{\eta} \varphi_{k}\right)^{2}=-q(\xi, \eta, \theta)
$$

и начальным данным

$$
\left.\varphi_{k}\right|_{\theta=0}=\stackrel{0}{\varphi}_{k}(\xi, \eta), \quad k=1,2 .
$$

ЛЕмма. Кривая l, образуемая совпадающими лучами исходных гармоник, выходящими из точки $\left(\xi_{0}, \eta_{0}\right)$, является многообразием локального резонанса в том смысле, что на ней выполнены резонансные соотношения $\nabla \varphi_{1}=\nabla \varphi_{2}, \quad(\xi, \eta, \theta) \in l$.

Справедливость этого утверждения проверяется непосредственными вычислениями.

Несобственные комбинационные фазы $\psi$ в главном члене отсутствуют, поэтому амплитуды $\stackrel{0}{B_{\psi}}$ при них тождественно равны нулю.

Собственные амплитуды $\stackrel{0}{A}$ главного члена ФАР являются гладкими функциями своих переменных и определяются из квазилинейной системы уравнений переноса

$$
i \partial_{\theta} \stackrel{0}{A_{k}}+2 i\left\langle\nabla \varphi_{k}, \nabla \stackrel{0}{A_{k}}\right\rangle+i \Delta \varphi_{k} \stackrel{0}{A_{k}}+\gamma\left[\left|0_{A}^{A}\right|^{2}+2 \sum_{m \neq k}\left|\stackrel{0}{A_{m}}\right|^{2}\right] \stackrel{0}{A_{k}}=0
$$

и начальных данных

$$
\left.\stackrel{0}{A_{k}}\right|_{\theta=0}=A_{k}(\xi, \eta), \quad k=1,2 .
$$

В первой поправке $U_{1}$ присутствуют несобственные комбинационные фазы $\psi \in \Psi_{1}=$ $\left(2 \varphi_{1}-\varphi_{2}, 2 \varphi_{2}-\varphi_{1}\right)$. Соответствуюшие им амплитуды $\stackrel{1}{B} \underset{\psi}{ }$ определяются из алгебраических соотношений

$$
l[\psi] \stackrel{1}{B}_{\psi}=\stackrel{1}{\mathbf{F}}_{\psi}, \quad \psi \in \Psi_{1} .
$$


Принимая во внимание лемму, непосредственными вычислениями проверяется, что множитель $l[\psi]$ на линии $l$ локального резонанса обрашается в нуль для всех комбинационных фаз первой поправки. Это приводит к тому, что все несобственные амплитуды ${ }_{B}$ имеют особенности на линии локального резонанса.

Для собственных амплитуд $\stackrel{1}{A}{ }_{k}(k=1,2)$ получаем неоднородную систему уравнений переноса. Правые части этой линейной системы имеют особенности на линии локального резонанса. Наличие особенностей объясняется тем, что в правую часть уравнений входят несобственные амплитуды $\stackrel{1}{B}$, первой поправки.

Для того чтобы проанализировать поведение амплитуд $\stackrel{1}{A}{ }_{k} \quad(k=1,2)$ при подходе к линии локального резонанса, удобно использовать переменные $D=\sqrt{S^{2}+R^{2}}$, $\Phi=\operatorname{arctg}(R / S)$, тем более что переход к этим переменным дает возможность получения равномерно пригодного выражения для асимптотики.

Система уравнений для определения собственных амплитуд первой поправки в переменных $(D, \Phi, \theta)$ записывается следуюшим образом:

$$
\partial_{\theta} A_{k}+a_{k} \partial_{D} A_{k}+\frac{1}{D} b_{k} \partial_{\Phi} A_{k}+c_{k} A_{1}+d_{k} A_{2}+e_{k} A_{1}^{*}+f_{k} A_{2}^{*}=\frac{1}{D^{2}} h_{k},
$$

начальные данные однородны

$$
\left.A_{k}\right|_{\theta=0}=0, \quad k=1,2 .
$$

Коэффициенты $a_{k}, b_{k}$ являются гладкими функциями и имеют порядок $O(D)$ при $D \rightarrow 0$. Справедливо следующее утверждение.

Теорема 2. Решение задачи (14), (15) для амплитуд первой поправки существует, единственно и при подходе к линии локального резонанса имеет асимптотику вида

$$
\stackrel{1}{A}_{k}(D, \Phi, \theta)=\sum_{m=-2}^{\infty} \stackrel{k}{\alpha}_{m}(\Phi, \theta) D^{m}, \quad D \rightarrow 0, \quad k=1,2, \quad \stackrel{k}{\alpha}_{-2} \not \equiv 0
$$

ДокАЗАТЕЛЬСтво теоремы проводится так же, как и в работе [8]. Выполняются формальные построения асимптотики, а затем проводится их обоснование.

Кроме исходных собственных фаз $\varphi_{1}$ и $\varphi_{2}$, в первой поправке имеется новая пара собственных фаз $\varphi$, отсутствующая в главном члене. Слагаемые, соответствующие этим фазам, должны компенсировать в начальных данных вклады от несобственных комбинационных фаз $\psi$.

Новые собственные фазы $\varphi$ определяются из уравнений эйконала (11) и начальных условий $\left.\varphi\right|_{\theta=0}=\left.\psi\right|_{\theta=0}$, где $\psi \in \Psi_{1}$. Соответствуюшие им амплитуды $\stackrel{1}{A}{ }_{\varphi}, \quad \neq \neq \varphi_{k}$, удовлетворяют однородным уравнениям типа (14) и начальным данным

$$
\left.\stackrel{1}{A}\right|_{\theta=0}=-\left.\stackrel{1}{B}_{\psi}\right|_{\theta=0}
$$




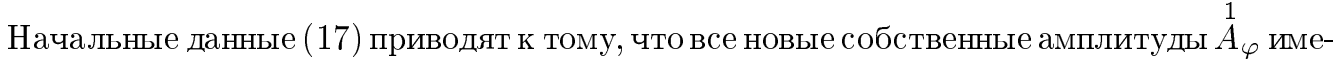
ют особенности при любых $\theta \geqslant 0$ на линии $l$ локального резонанса из-за особенностей соответствующих $\stackrel{1}{B}$.

В следующей поправке увеличивается число как несобственных комбинационных фаз $\psi$, так и компенсируюших их новых собственных фаз $\varphi$. Порядок особенностей при подходе к линии локального резонанса у всех амплитуд второй поправки увеличивается до $O\left(D^{-4}\right)$. Представление (8) в окрестности $l$ становится непригодным. Здесь предлагается использовать внутренние "растянутые" переменные $\sigma=S / \sqrt{\varepsilon}, \rho=R / \sqrt{\varepsilon}$ и решение исходной задачи искать в виде анзаца (9). При этом в представлении (9) сохраняется только одна фаза, т.к. в окрестности линии $l$ фазы исходных гармоник отличаются на величину порядка $O\left(D^{2}\right), D \rightarrow 0$.

Для амплитуды $\stackrel{0}{V}$ главного члена внутреннего $\Phi$ АР получаем двумерное нелинейное уравнение Шредингера

$$
i \partial_{\theta} v+a(\theta) \partial_{\sigma}^{2} v+b(\theta) \partial_{\sigma \rho}^{2} v+c(\theta) \partial_{\rho}^{2} v+f(\theta) v+\gamma|v|^{2} v=0
$$

и начальное условие

$$
\left.v\right|_{\theta=0}=A+B \exp \left\{i\left(k_{1} \sigma^{2}+k_{2} \rho^{2}\right)\right\} .
$$

Справедлива следуюшая теорема.

ТеОрема 3. Для любого $N$ имеется такое число $T_{1}(N)$, что для всех $\theta \in\left[0, T_{1}\right]$ решение задачи (18), (19) существует, единственно и при $\sigma^{2}+\rho^{2} \rightarrow \infty$ обладает равномерной по $\theta$ асимптотикой вида

$$
\begin{aligned}
v(\sigma, \rho, \theta)=\stackrel{0}{v}(\theta) & +\stackrel{0}{v}_{\nu}(\theta) \exp \left\{i\left(\kappa_{1}(\theta) \sigma^{2}+\kappa_{2}(\theta) \rho^{2}\right)\right\}+ \\
& +\sum_{m \geqslant 1}^{N} \frac{1}{\left(\sigma^{2}+\rho^{2}\right)^{m}}\left(-{ }_{v}^{2 m}(\rho, \sigma, \theta)+\right. \\
& \left.+\sum_{\nu}^{-2 m}{ }_{v}(\rho, \sigma, \theta) \exp \left\{i\left(\kappa_{1}^{\nu}(\theta) \sigma^{2}+\kappa_{2}^{\nu}(\theta) \rho^{2}\right)\right\}\right)+ \\
& +O\left(\left(\sigma^{2}+\rho^{2}\right)^{-(N+1)}\right)
\end{aligned}
$$

Вид асимптотики (20) определяется из условия согласования с уже построенным внешним $\Phi$ АР.

ДОКАЗАТЕЛЬСТВО теоремы проводится с использованием пребразования Фурье по пространственным переменным [9]. Точное решение ишется в виде суммы нескольких первых членов асимптотики и остатка. После преобразования Фурье для остатка выписывается интегродифференциальное уравнение, которое можно рассматривать как уравнение в некотором банаховом пространстве. Выбор пространства осуществляется из соображений ограниченности получаемых операторов [10]. Таким пространством оказывается пространство $\mathcal{M}_{r}$ с введенной в нем нормой графика замкнутого оператора 
свертки с быстрой экспонентой. Пользуясь этим и липшицевостью полученных операторов, методом последовательных приближений доказывается сушествование решения уравнения для остатка в классе ограниченных функций.

Старшие поправки $\stackrel{n}{V}, n=1,2$, находятся из задач Коши для уравнений Шредингера, линеаризованных на амплитудах предыдуших приближений. Начальные данные для этих уравнений получаются из условия (6) после перехода к "внутренним" переменным $\sigma$ и $\rho$ и соответствующего разложения полученных функций в ряд по малому параметру.

Эти построения завершают доказательство теоремы 1.

\section{Список литературы}

[1] M. Ablowitz, A. Funk, A. Newell. Stud. Appl. Math. 1973. V. 52. P. 51-74.

[2] J. Kevorkian. SIAM J. Appl. Math. 1971. V. 20. P. 364-373.

[3] L. Rubenfeld. Stud. Appl. Math. 1977. V. 57. P. 77-92.

[4] J. C. Neu. SIAM J. Appl. Math. 1983. V. 43. № 1. P. 141-156.

[5] С. Ю. Доброхотов, П. Н. Жевандров, В. М. Кузьмина. Прикл. матем. и мех. 1987. Т. 51. № 5. C. $798-806$.

[6] Л. А. Калякин. Метод ВКБ в слабо нелинейной задаче с локальным резонансом. В сб.: Асимптотические методы решения задач математической физики. Ред. В. Ю. Новокшенов и др. Уфа: Изд-во БНЦ УрО АН СССР, 1989. С. 56-69.

[7] Л. А. Калякин. Мат. заметки. 1988. Т. 44. № 5. С. 697-699.

[8] С. Г. Глебов. Дифф. уравнения. 1995. Т. 31. № 8. С. 1402-1408.

[9] С. Г. Глебов. Теорема существования для $N$-мерного нелинейного уравнения Шредингера. В сб.: Асимптотики и симметрии в нелинейных динамических системах. Ред. Л. А. Калякин. Уфа: Изд-во Института математики с Вычислительным центром РАН, 1995. С. 31-37.

[10] Л. А. Калякин, С. Г. Глебов. Мат. заметки. 1994. Т. 56. С. 32-40. 